\title{
Quenching of Meridional Circulation in Flux Transport Dynamo Models
}

\author{
Bidya Binay Karak Arnab Rai Choudhuri
}

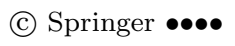

\begin{abstract}
Guided by the recent observational result that the meridional circulation of the Sun becomes weaker at the time of the sunspot maximum, we have included a parametric quenching of the meridional circulation in solar dynamo models such that the meridional circulation becomes weaker when the magnetic field at the base of the convection zone is stronger. We find that a flux transport solar dynamo tends to become unstable on including this quenching of meridional circulation if the diffusivity in the convection zone is less than about $2 \times 10^{11} \mathrm{~cm}^{2} \mathrm{~s}^{-1}$. The quenching of $\alpha$, however, has a stabilizing effect and it is possible to stabilize a dynamo with low diffusivity with sufficiently strong $\alpha$-quenching. For dynamo models with high diffusivity, the quenching of meridional circulation does not produce a large effect and the dynamo remains stable. We present a solar-like solution from a dynamo model with diffusivity $2.8 \times 10^{12} \mathrm{~cm}^{2} \mathrm{~s}^{-1}$ in which the quenching of meridional circulation makes the meridional circulation vary periodically with solar cycle as observed and does not have any other significant effect on the dynamo.
\end{abstract}

\section{Introduction}

Flux transport dynamo model (Choudhuri, Schüssler, and Dikpati 1995: Durney 1995 Dikpati and Charbonneau 1999| Nandy and Choudhuri 2002 Chatterjee, Nandy, and Choudhuri 2004 Charbonneau 2010; Choudhuri 2011) is the most promising model of solar cycle at present. In this model, the strong toroidal field is generated near the base of the convection zone due to the stretching of the poloidal field by the strong differential rotation and the poloidal field is generated near the surface through the Babcock-Leighton mechanism. The meridional circulation and the turbulent diffusivity are the two important flux transport agents from the source of the poloidal field (near the surface) to the source region of the toroidal field (bottom of the convection zone). The meridional circulation plays a very crucial role in the flux transport dynamo model by determining its period (Dikpati and Charbonneau 1999) and also has an effect on the cycle strength (Yeates, Nandy, and Mackay 2008; Karak 2010). It appears that the meridional circulation is crucial in modeling 
many irregular features of the solar cycle like the Waldmeier effect (Karak and Choudhuri 2011) and the Maunder-like grand minima (Karak 2010). Moreover, Passos and Lopes (2008; see also Lopes and Passos 2009a) found the importance of meridional circulation when they studied the solar cycle using a low-order dynamo model.

Our observational knowledge of the meridional circulation is very limited at the present time. It is poleward near the surface with an amplitude of around 20 $\mathrm{m} \mathrm{s}^{-1}$. However, the detailed profile of the meridional circulation and the return flow at the bottom of the convection zone are not observationally established yet. It is believed that the meridional circulation is caused by the combined effect of buoyancy, Reynolds stresses, latitudinal pressure gradient and the Coriolis force acting on the mean zonal flow (Kitchatinov and Rüdiger 1995). Therefore we expect that there may be random variations in this flow due to fluctuations in any of these driving forces. From the periods of various past cycles, Karak and Choudhuri (2011) have attempted to draw some conclusions about the random variations in the meridional circulation (also see Passos and Lopes 2008; Lopes and Passos 2009b). In addition to these random variations, there must also be a periodic variation with the solar cycle due to the feedback of the Lorentz force of the dynamo-generated magnetic field. This type of periodic variation has recently been reported by Hathaway and Rightmire (2010) and Basu and Antia (2010), who found that the circulation speed was slightly weaker at the time of the sunspot maximum. Such a thing was also seen in a simulation of flux transport dynamo (Rempel 2006). The main aim of this paper is to study the effects of the periodic variation of meridional circulation on the flux transport dynamo model. We produce the periodic variation of meridional circulation by introducing a simple quenching of meridional circulation by the dynamo-generated magnetic field. Our main conclusion is that the quenching of the meridional circulation has very different effects on flux transport dynamo models using different values of the turbulent diffusivity.

During the last few years, two classes of flux transport dynamo models have been developed in considerable details: (i) the high-diffusivity model in which the value of the turbulent diffusivity $\eta$ in the convection zone is taken in the range $\sim 10^{12}-10^{13} \mathrm{~cm}^{2} \mathrm{~s}^{-1}$ (Chatterjee, Nandy and Choudhuri 2004; Choudhuri, Chatterjee and Jiang 2007) and (ii) the low-diffusivity model in which $\eta$ in the convection zone is $\sim 10^{10}-10^{11} \mathrm{~cm}^{2} \mathrm{~s}^{-1}$ (Dikpati and Charbonneau 1999; Dikpati and Gilman 2006). We may note that the simple mixing length theory gives the value of $\eta \sim 1-4 \times 10^{12} \mathrm{~cm}^{2} \mathrm{~s}^{-1}$ (Parker 1979, p. 629), similar to what is used in the high-diffusivity model. We find that the quenching of meridional circulation does not have any significant effect on the dynamo when the diffusivity is high. However, when the diffusivity is low, the dynamo model becomes unstable on introducing the quenching of meridional circulation unless we also introduce a strong quenching of $\alpha$ to stabilize the solution. We show that the physics of what is happening can be understood on the basis of some results presented by Yeates, Nandy and Mackay (2008). It may be mentioned that several authors have recently argued in favour of the high-diffusivity model (Chatterjee and Choudhuri 2006; Jiang, Chatterjee and Choudhuri 2007; Goel and Choudhuri 2009; Choudhuri and Karak 2009; Hotta and Yokoyama 2010a, 2010b; Karak 2010; Karak and Choudhuri 2011; Kitchatinov and Olemskoy 2011). The results of this paper provide further support to the high-diffusivity model. 


\section{Model}

In the flux transport dynamo model, the evolution of magnetic fields are governed by the following two equations:

$$
\begin{gathered}
\frac{\partial A}{\partial t}+\frac{1}{s}(\mathbf{v} \cdot \nabla)(s A)=\eta_{p}\left(\nabla^{2}-\frac{1}{s^{2}}\right) A+S(r, \theta ; B) \\
\frac{\partial B}{\partial t}+\frac{1}{r}\left[\frac{\partial}{\partial r}\left(r v_{r} B\right)+\frac{\partial}{\partial \theta}\left(v_{\theta} B\right)\right]=\eta_{t}\left(\nabla^{2}-\frac{1}{s^{2}}\right) B+s\left(\mathbf{B}_{p} . \nabla\right) \Omega+\frac{1}{r} \frac{d \eta_{t}}{d r} \frac{\partial(r B)}{\partial r},
\end{gathered}
$$

where $s=r \sin \theta$. Here $\mathbf{B}_{\mathbf{p}}$ correspond to the poloidal components of magnetic field which is given by the curl of $A(r, \theta, t) \mathbf{e}_{\phi}$, whereas $B(r, \theta, t)$ correspond to the toroidal components of magnetic field. $\mathbf{v}$ is the velocity of the meridional flow. The term $S(r, \theta ; B)$ is the responsible for generating the poloidal field, whereas $\Omega$ is the internal angular velocity of the Sun, and $\eta_{p}, \eta_{t}$ are the turbulent diffusivities for the poloidal and toroidal fields respectively.

For a particular solar dynamo model, we need to specify the values of the various parameters, such as $\Omega, \mathbf{v}, \eta_{p}, \eta_{t}$ and $S(r, \theta ; B)$. Some of our calculations are done by using exactly same parameters as used by Dikpati and Charbonneau (1999) in their 'reference solution' except for the value of $u_{0}$ which is taken as $20 \mathrm{~m} \mathrm{~s}^{-1}$ rather than $10 \mathrm{~m} \mathrm{~s}^{-1}$ as quoted by them. Only with this value of $u_{0}$, we are able to reproduce their results (Jiang, Chatterjee, and Choudhuri 2007). To see the effect of quenching of meridional circulation on this model, we introduce the quenching as described in Section 3. We also have to suppress the $\alpha$-quenching to some extent, in order to see the effect of meridional circulation quenching clearly, as discussed in Section 4. One of the advantages of this model is that it gives periodic solutions even when the diffusivity is increased by a few orders. To understand how the results change on changing the diffusivity, we do runs with different diffusivity while keeping most of the other things constant. Note that in this model a single value of magnetic turbulent diffusivity for the toroidal and the poloidal field is used. We call this dynamo model the DC99 in spite of some differences with the original 'reference solution' of Dikpati and Charbonneau (1999). Our main conclusion in $\S 4$ will be that the quenching of meridional circulation can make this model unstable if the diffusivity in the solar convection zone is assumed to be lower than about $2 \times 10^{11} \mathrm{~cm}^{2} \mathrm{~s}^{-1}$, provided we do not have a strong $\alpha$-quenching to stabilize the system.

One solar dynamo model which has been developed and used extensively in our group is the model of Chatterjee, Nandy and Choudhuri (2004), who presented what they called their 'standard model'. We, however, recently used slightly different values of a few parameters which are discussed in Karak (2010). We call this K10 model. Karak (2010) had used a rather high diffusivity of $2.8 \times 10^{12} \mathrm{~cm}^{2} \mathrm{~s}^{-1}$. If we change the diffusivity to a much lower value keeping the other parameters of this model constant, we find that this model does not give periodic solutions. So we present results of the meridional circulation quenching on the K10 model only for the high diffusivity and the model is found to be stable like the high-diffusivity version of the DC99 model. 


\section{Quenching of Meridional Circulation}

We have reliable data of the temporal variation of the surface meridional circulation of the last one cycle only (Hathaway and Rightmire 2010; Basu and Antia 2010). Therefore, in most of our earlier kinematic calculations, we had used a constant value of meridional circulation. It is easy to show that a toroidal magnetic field at the base of the convection zone will have a poleward Lorentz force (van Ballegooijen and Choudhuri 1988). During the solar maximum, when the toroidal field at the base of the convection zone is particularly strong, we expect that the poleward Lorentz force due to it will oppose the meridional circulation which is equatorward there and thereby would lead to a reduction in the meridional circulation at the time of the solar maximum. One needs to consider this feedback of the magnetic field on the meridional circulation while studying the solar cycle using kinematic dynamo models. For a full treatment of the problem, it is necessary to solve the Navier-Stokes equation for the meridional circulation and to carefully address such questions as to how much time will be required for the meridional circulation to slow down at the surface after the poleward Lorentz force starts acting at the base of the convection zone. We are carrying out a detailed investigation of some of these issues, which will be presented in a future paper. In this paper, we present results of dynamo calculations obtained with the simplistic assumption that meridional circulation throughout the convection zone gets reduced at the solar maximum because of a quenching due to the magnetic field. Therefore we introduce a quenching in the amplitude of the meridional circulation in the following form:

$$
v_{0}=v_{0}^{\prime} /\left[1+\left(\bar{B} / B_{0}^{\prime}\right)^{2}\right]
$$

where $\bar{B}$ is the average toroidal field in the tachocline $\left(r=0.65 R_{\odot}\right.$ to $\left.0.71 R_{\odot}\right)$. Although the above form of the quenching seems very simple, it does a very good job in reducing the amplitude of meridional circulation $v_{0}$ rapidly once the toroidal field approaches the value $B_{0}^{\prime}$ and produces some striking effects on the behaviour of the flux transport dynamo - especially if the diffusivity is low. We may also mention that although the meridional circulation and the angular velocity are related, we are not considering any quenching on the angular velocity in this work.

\section{Results}

We study the effect of meridional circulation quenching on both the DC99 and K10 models as mentioned in Section 2. For both the models, we first run the code for several solar cycles without the quenching in the meridional circulation. Then we stop the code at some point and introduce the quenching on meridional circulation using Equation (3). After this change in meridional circulation, we run the code again for several solar cycles. For the DC99 model, we are able to do calculations by varying diffusivity over a wide range. For the K10 model, however, we present results only for high diffusivity, since this model does not give periodic solutions for low diffusivity. 


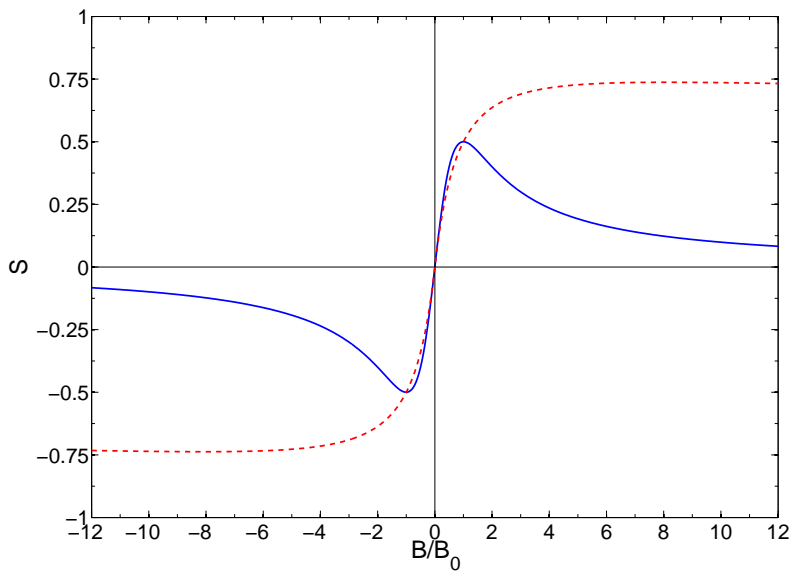

Figure 1. The poloidal field generation factor $S(r, \theta ; B)$ as a function of the toroidal field. The quenching profile shown by the solid line was used in Dikpati and Charbonneau (1999). The profile shown by the dashed line is used in most of the calculations in this paper.

\subsection{Results from the DC99 model}

As we pointed out in Section 2, we use the model of Dikpati and Charbonneau (1999) apart from changing the value of $u_{0}$ they quoted. We make another crucial change. It is known for a long time that the $\alpha$-quenching has a stabilizing effect on the dynamo - see Section 1 of Choudhuri (1992). Dikpati and Charbonneau (1999) had used a rather strong $\alpha$-quenching given by

$$
\alpha=\alpha_{0} /\left[1+\left(B / B_{0}\right)^{2}\right] .
$$

This makes the source function $S(r, \theta ; B)=\alpha B$ in (1) vary as a function of $B$ as shown by the solid line in Figure 1 . Such a strong $\alpha$-quenching would suppress any possible instability induced by the quenching of meridional circulation. In order to see the effects of meridional circulation quenching, it is essential to make the $\alpha$-quenching weaker. We present calculations obtained with a milder $\alpha$-quenching given by

$$
\alpha=\alpha_{0} /\left[1+\left(|B| / B_{0}\right)^{1.1}\right] .
$$

and shown by the dashed line in Figure 1 . Without any $\alpha$-quenching at all, $S(r, \theta ; B)$ which is equal to $\alpha B$ would simply increase linearly with $B$. We shall give arguments below why we consider the $\alpha$-quenching used by Dikpati and Charbonneau (1999) to be unrealistically strong. It may be noted that, before we introduce the quenching in meridional circulation, the $\alpha$-quenching is the only source of nonlinearity in the problem and determines the amplitude of the magnetic field. If we increase the numerical value of $B_{0}$ in the code by a factor $s$, the magnetic field in the solution everywhere increases uniformly by the same factor $s$.

Figure 2 shows the results of meridional circulation quenching on the DC99 model using the same low diffusivity of $5 \times 10^{10} \mathrm{~cm}^{2} \mathrm{~s}^{-1}$ as used by Dikpati and 


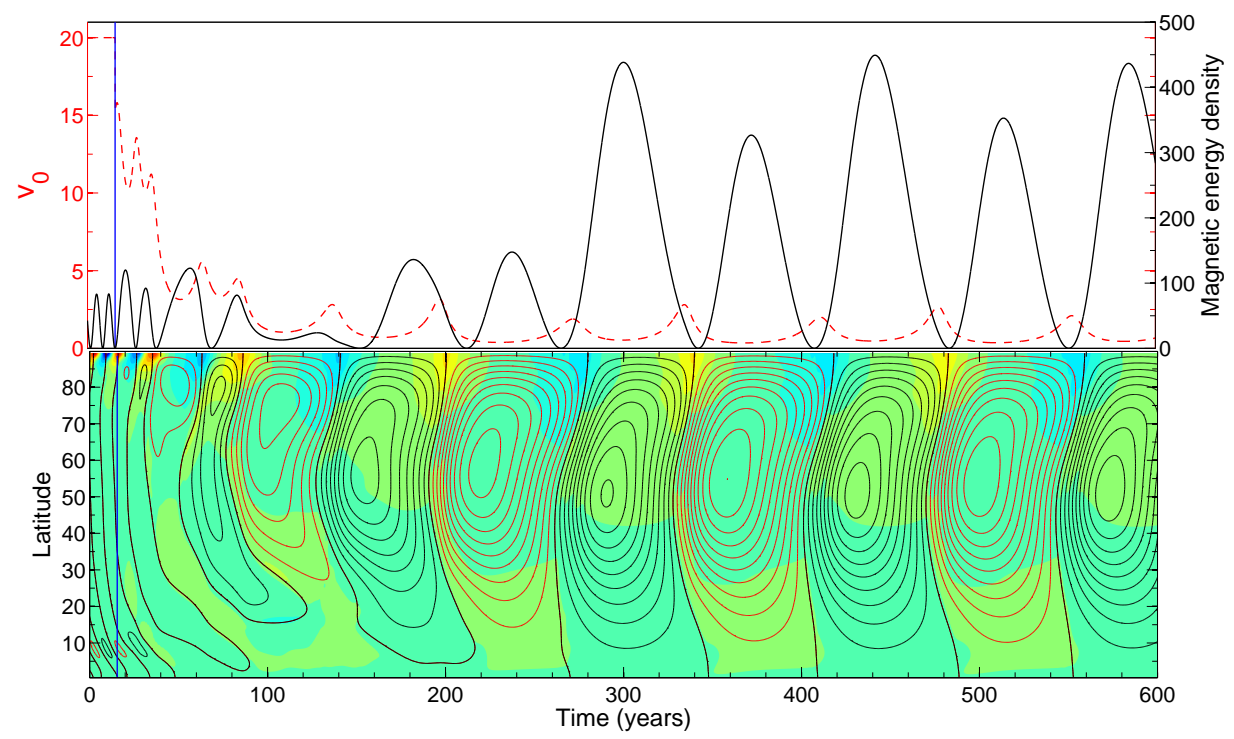

Figure 2. Results from DC99 model: Upper panel shows the variation of the amplitude of meridional circulation $v_{0}$ (dashed line) and the magnetic energy density of the toroidal field at latitude $15^{\circ}$ at the bottom of the convection zone (solid line). The vertical solid line indicates the time of the initiation of quenching. Lower panel shows the butterfly diagram of the toroidal field (contours). The background shows the weak diffuse radial field on the solar surface. The diffusivity used in this case is $5 \times 10^{10} \mathrm{~cm}^{2} \mathrm{~s}^{-1}$.

Charbonneau (1999). We have used a rather weak quenching of meridional circulation in which $B_{0}^{\prime}$ appearing in (3) is taken to be $B_{0}^{\prime} / B_{0}=9.4$. Note that a larger value of $B_{0}^{\prime} / B_{0}$ implies a weaker quenching of meridional circulation. The upper panel shows the variation of the $v_{0}$ (dashed line) and the magnetic energy density of the toroidal field at latitude $15^{\circ}$ at the bottom of the convection zone (solid line). The vertical solid line indicates the time when the quenching in meridional circulation in accordance with (3) is included. We see that immediately after the commencement of the quenching the value of $v_{0}$ drops to around $16 \mathrm{~m} \mathrm{~s}^{-1}$ from the usual value $20 \mathrm{~m} \mathrm{~s}^{-1}$ and then it oscillates with the solar cycle. However, the value of $v_{0}$ keeps decreasing with time and falls close to zero in about four to five solar cycles. Then it oscillates with the solar cycle remaining close to the zero value. We also note that the cycle period increases as $v_{0}$ decreases, which is expected in a flux transport dynamo. In the butterfly diagram given in the bottom panel, we see that the equatorial propagation of the toroidal field does not happen beyond a few solar cycles after the commencement of the quenching. This is because the meridional circulation which is responsible for this equatorial propagation of the toroidal field (Choudhuri, Schüssler, and Dikpati 1995) has become very weak. It is clear that the low-diffusivity model fails to give solar-like oscillation after the inclusion of the quenching in meridional circulation.

Next, Figure 3 shows the results on increasing the diffusivity of the DC99 model to the value $2.8 \times 10^{12} \mathrm{~cm}^{2} \mathrm{~s}^{-1}$ (which is used in the K10 model). When 


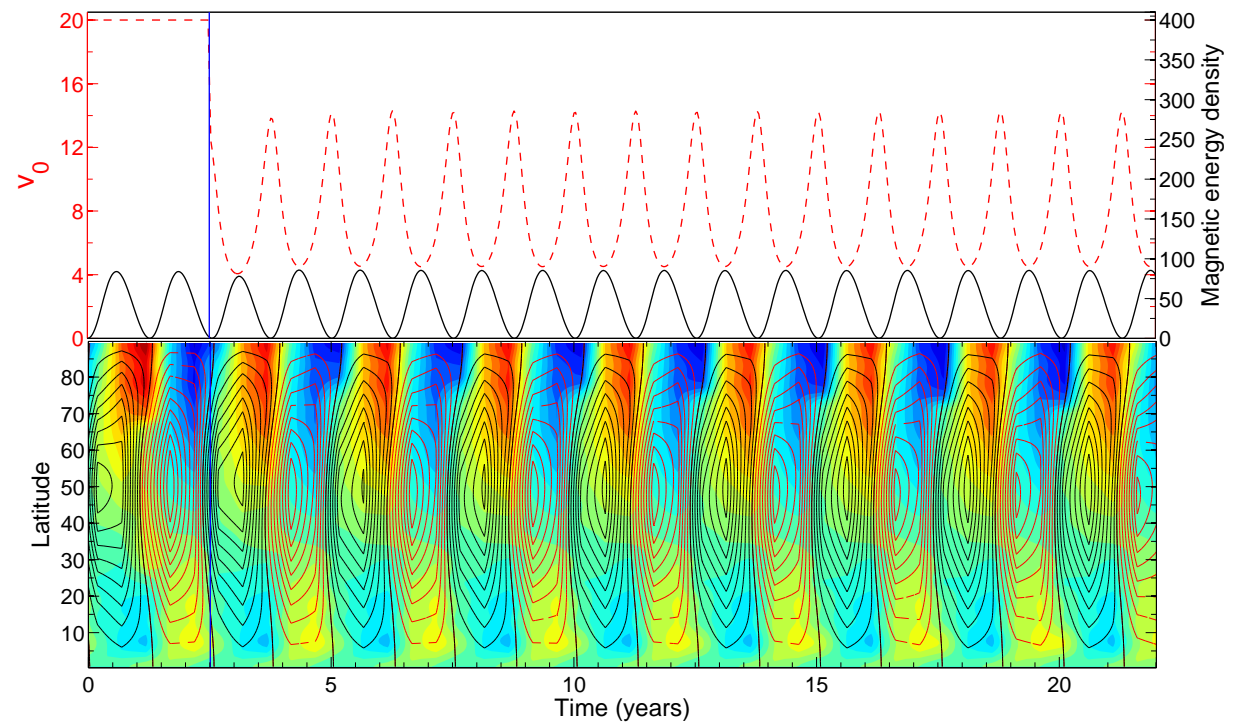

Figure 3. Same as Figure 2 but in this case the diffusivity is increased to $2.8 \times 10^{12} \mathrm{~cm}^{2} \mathrm{~s}^{-1}$.

the diffusivity is increased, we need to increase the value of $\alpha$ along with it, otherwise the solutions decay away. While the value of $\alpha_{0}$ used to generate Figure 2 was $0.2 \mathrm{~m} \mathrm{~s}^{-1}$ the same as what was used in the original work of DC99, we now take $\alpha_{0}$ to be $215 \mathrm{~m} \mathrm{~s}^{-1}$. Except the changes in diffusivity and $\alpha_{0}$, all the other parameters remain the same between Figures 2 and 3. Figure 3 plots the same things as Figure 2, for an increased diffusivity. We find that now we have a stable periodic solution, although the butterfly diagram does not look solar-like and the period is very short. If we run the code without the quenching of meridional circulation, then also the butterfly diagram looks very much like what we see in Figure 3, indicating that this quenching does not have too much effect when the diffusivity is high.

To understand how the nature of the solution changes from Figure 2 to Figure 3, we have made runs for different values of diffusivity. When increasing diffusivity, we have also increased $\alpha_{0}$ in such a way that the toroidal field comes out to have very similar strengths in all the runs. The upper panel of Figure 4 shows the values of $\alpha_{0}$ chosen for different values of diffusivity. The lower panel of Figure 4 shows how other important properties of the solution change of changing diffusivity. One of the things shown is how the period of the eventual relaxed solution changes on changing the diffusivity. For any diffusivity, the amplitude of meridional circulation oscillates between a maximum and a minimum value, as we see in Figures 2 and 3. In the lower panel of Figure 4, we also plot the maximum and minimum values of the meridional circulation for different diffusivities. When the diffusivity is low, as in the case of Figure 2, the quenching of meridional circulation makes the system unstable so that it 


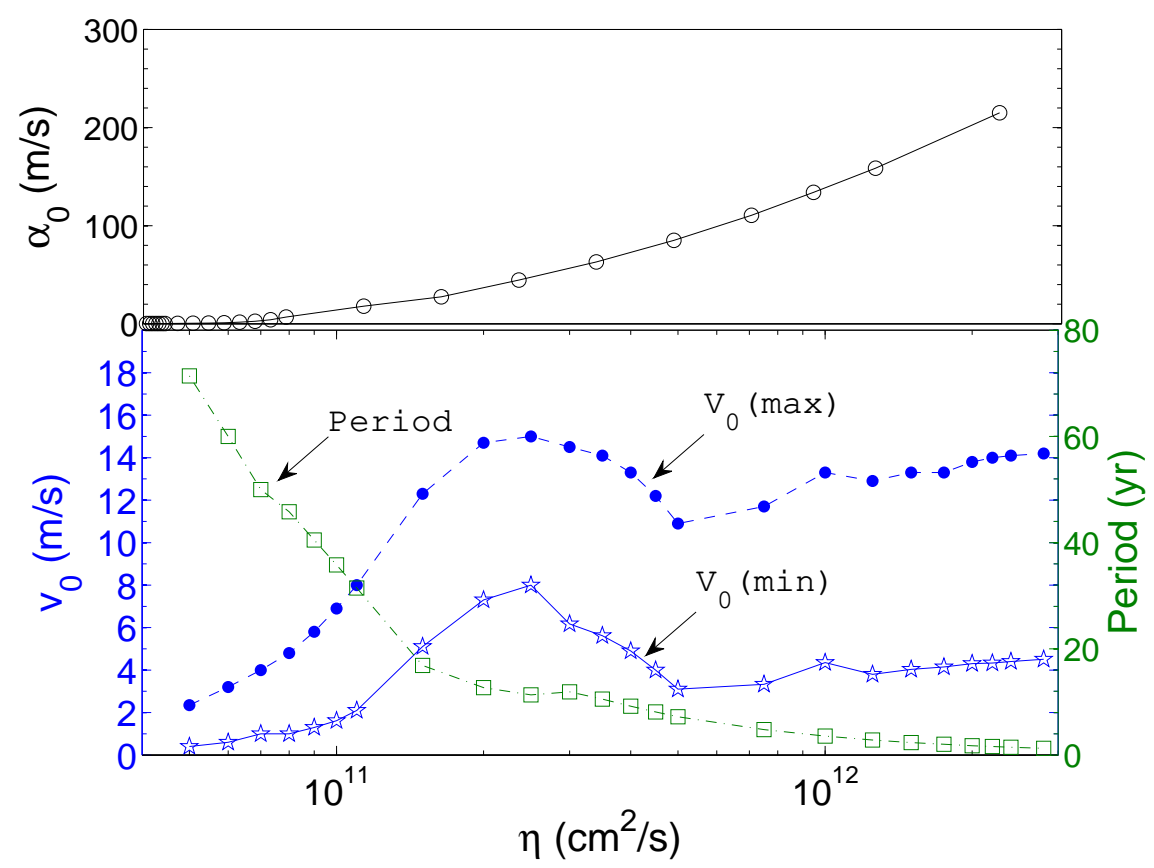

Figure 4. Upper panel shows the values of $\alpha_{0}$ required to get the stable solution when we change the diffusivity $\eta$ (shown along the horizontal axis). Lower panel shows the variation of cycle period (square symbol), maximum $v_{0}$ (filled circle) and the minimum $v_{0}$ (star symbol) of the relaxed solutions as a function of diffusivity.

eventually relaxes to a solution in which the meridional circulation falls to a very low value and the period is large. On the other hand, when the diffusivity is high, the solution has an asymptotic low value of period whereas meridional circulation remains much stronger. The maximum value of meridional circulation in the high-diffusivity limit is essentially the value that we would see when the quenching of meridional circulation is switched off and the effect of quenching is not significant when diffusivity is high. Figure 4 makes it clear that the transition from instability to stability is not a very sharp transition, but takes place roughly around the value $2 \times 10^{11} \mathrm{~cm}^{2} \mathrm{~s}^{-1}$ of diffusivity.

The reason for this kind of peculiar behaviour can be understood from the analysis of Yeates, Nandy, and Mackay (2008). When $v_{0}$ decreases due to the quenching, the cycle period is longer and the poloidal field spends more time in the convection zone. This will result in two opposing effects. On the one hand, diffusion gets more time to act on the poloidal field and the toroidal field ultimately produced from this weaker poloidal field will tend to be weaker. On the other hand, the differential rotation also gets more time to generate the 
toroidal field, thereby tending to make the toroidal field stronger. Whether the toroidal field will finally be weaker or stronger will depend on which of these two competing effects wins over. When the diffusivity is high, the diffusion of the fields is more important and the toroidal field is weaker when the meridional circulation decreases due to the quenching because diffusion has more time to act on the fields. This decrease of the toroidal field will make the meridional circulation stronger according to Equation (3). Thus the meridional circulation will try to bounce back to a higher value. In this way, this model stabilizes under the quenching of the meridional circulation. However, if the diffusivity is low, things are opposite. When the meridional circulation decreases due to quenching of the strong toroidal field and the dynamo period increases, the differential rotation generating the toroidal field for a longer time is the dominant effect rather than diffusion. Then the toroidal field becomes stronger and this decreases the meridional circulation further according to Equation (3). This leads to a runaway unstable situation until the meridional circulation drops to very low values. These arguments explain the results seen in Figures 2 and 3.

We also made some runs by making $B_{0}^{\prime} / B_{0}$ lower than 9.4 used to generate Figures 2-4, i.e. by making the quenching of meridional circulation stronger. Then the fall in the value of $v_{0}$ after introducing the quenching was faster than what is seen in Figure 2 and the eventual periods were longer. Otherwise, the qualitative behaviour of the system does not change when $B_{0}^{\prime} / B_{0}$ is varied between 1 and 10 .

As we pointed out, we have used a much weaker $\alpha$-quenching in our calculations (as indicated by the dashed curve in Figure 1) than what Dikpati and Charbonneau (1999) had used. When we repeated our calculations by using the stronger $\alpha$-quenching of Dikpati and Charbonneau (1999) shown by the solid curve in Figure 1, we found that the dynamo solutions do not change much on introducing the quenching of the meridional circulation even when the diffusivity is low. In other words, the strong $\alpha$-quenching suppresses the instability induced by the quenching of the meridional circulation. It is not difficult to understand physically what is happening. The strong $\alpha$-quenching does not allow the magnetic fields to grow the way they would grow in its absence and thus the tendency of runaway growth of the toroidal field on introducing the quenching of the meridional circulation in the low-diffusivity case is stabilized.

Since Figure 2 does not agree with the behaviours of solar magnetic fields, one is tempted to conclude that the solar dynamo could not be a low-diffusivity dynamo - unless there is a stabilizing effect due to a strong $\alpha$-quenching. So it is a crucial question whether a strong $\alpha$-quenching is expected in the Sun. The Babcock-Leighton mechanism, which is parameterized by $\alpha$, depends on the tilts of active regions. These tilts are produced by the Coriolis force acting on the rising flux tubes and the effect of the Coriolis force certainly does become weaker when the magnetic field is stronger (Choudhuri 1989; D'Silva and Choudhuri 1993). However, magnetic fields of different sunspots do not vary too much, suggesting that the toroidal field probably becomes buoyant when it reaches a critical value and rising flux tubes within the convection zone may not have widely different values of the magnetic field. More generation of toroidal field probably means more active regions and not stronger magnetic fields within 
individual active regions. If that is the case, then we do not think that one can invoke a strong $\alpha$-quenching to stabilize the dynamo. In spite of many uncertainties in our present understanding of the solar dynamo and $\alpha$-quenching, it seems unlikely that the solar dynamo is a low-diffusivity dynamo which has a tendency of becoming unstable on introducing the quenching of the meridional circulation.

\subsection{Results from K10 model}

Let us now present the results from the K10 model, which uses a high diffusivity $2.8 \times 10^{12} \mathrm{~cm}^{2} \mathrm{~s}^{-1}$ and produces solar-like solutions. We saw above that the DC model with such a high diffusivity remains impervious to the effects of meridional circulation quenching, but the solutions do not look solar-like. Now we find that the K10 model, which uses this high diffusivity and produces solar-like solutions, also does not change qualitatively on introducing the quenching of meridional circulation. As in the case of Figures 2 and 3, the dashed (red) line in Figure 5 shows the variation of $v_{0}$ whereas the solid (black) line shows the variation of the magnetic energy density of the toroidal field at latitude $15^{\circ}$ at the bottom of the convection zone (a measure of sunspot number). The vertical solid line indicates the time of introduction of the quenching. We see from this figure that, soon after introducing the quenching, the value of $v_{0}$ drops to around $24 \mathrm{~m} \mathrm{~s}^{-1}$ from the usual value $30 \mathrm{~m} \mathrm{~s}^{-1}$. Then $v_{0}$ oscillates with the solar cycle, becoming weaker when the toroidal field becomes strong and vice versa. Since the results obtained from the K10 model can be compared with observations, it may be noted that the amplitude variation of the $v_{0}$ is comparable to observational plot shown in Figure 4 of Hathaway and Rightmire (2010). We achieved this by suitably adjusting the parameter $B_{0}^{\prime}$ appearing in (3). When the quenching is not present, the only nonlinearity in our model comes from magnetic buoyancy, which is treated by allowing the toroidal field to erupt whenever its value crosses a critical value $B_{c}$ within the convection zone (Chatterjee, Nandy and Choudhuri 2004). This limits the growth of the dynamo and makes the strongest toroidal fields at the bottom of the convection zone hover around $B_{c}$. It is $B_{c}$ which determines the scale of the magnetic field in K10 model just as $B_{0}$ determines the scale of the magnetic field in the DC99 model. We have used $B_{0}^{\prime} / B_{c}=1.0 / 0.8$ which make the variation of the meridional circulation comparable to what is observed.

The bottom panel of Figure 5 shows the butterfly diagram of the sunspot eruptions along with the radial field on the surface. We point out that we have chosen the value of $v_{0}^{\prime}$ (which is same as $v_{0}$ in Karak 2010) in such a way that after the addition of quenching the period of the solar cycle becomes close to 11 year. We see that the butterfly diagram with the inclusion of the quenching of meridional circulation looks very similar to the butterfly diagrams without such quenching as presented by Chatterjee, Nandy and Choudhuri (2004). We may point out that the high-diffusivity solution from the DC99 model shown in Figure 3, apart from not producing solar-like butterfly diagrams, had a rather small period. We believe that the main reason behind this small period is that magnetic buoyancy was treated differently in this model. See Choudhuri, Nandy, 


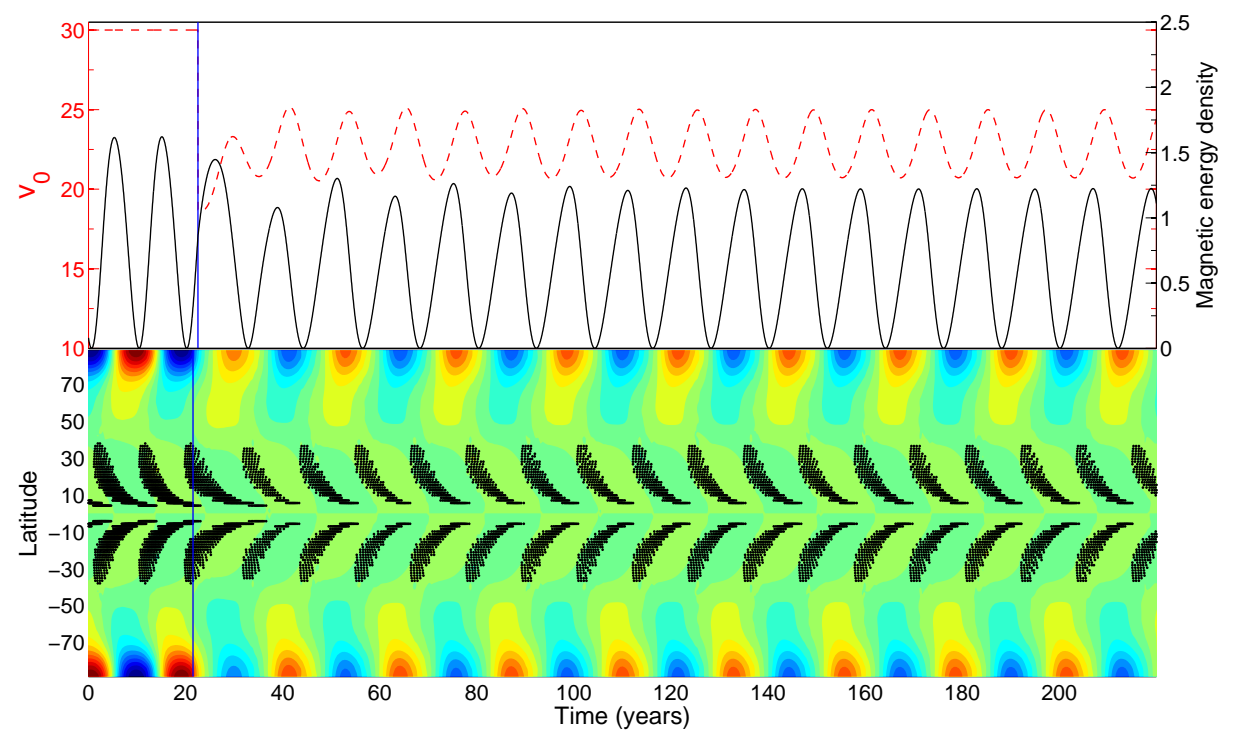

Figure 5. Results from K10 model: Upper panel shows the variation of the amplitude of meridional circulation $v_{0}$ (dashed line) and the magnetic energy density of the toroidal field at latitude $15^{\circ}$ at the bottom of the convection zone (solid line). The vertical solid line indicates the time of the initiation of quenching. Lower panel shows the butterfly diagram of the sunspot eruptions. The background shows diffuse radial field on the solar surface.

and Chatterjee (2005) for a discussion of how the period of the dynamo can be very different on treating magnetic buoyancy differently, even when the other parameters are kept the same.

Finally, since we introduced stochastic fluctuations in the poloidal field generation process in some of our calculations, one last question we address is whether dynamo models with stochastic fluctuations behave differently on introducing the quenching of meridional circulation. The result is presented in Figure 6. In this case, the amplitude of $\alpha$ is changed after the coherence time 6 months, the level of fluctuations being $133 \%$. We find that now there are some irregularities in the cycles as we would expect and as we find in the calculations without including the quenching in the meridional circulation.

Our conclusion is that, in the case of the high-diffusivity K10 model, the results remain qualitatively similar whether we include the quenching in the meridional circulation or not.

\section{Conclusion}

We have considered the Lorentz feedback of the dynamo-generated magnetic field on the meridional circulation. We first performed numerical experiments on the DC99 dynamo model, varying the diffusivity from the rather low value originally used by Dikpati and Charbonneau (1999) to values nearly two orders of 


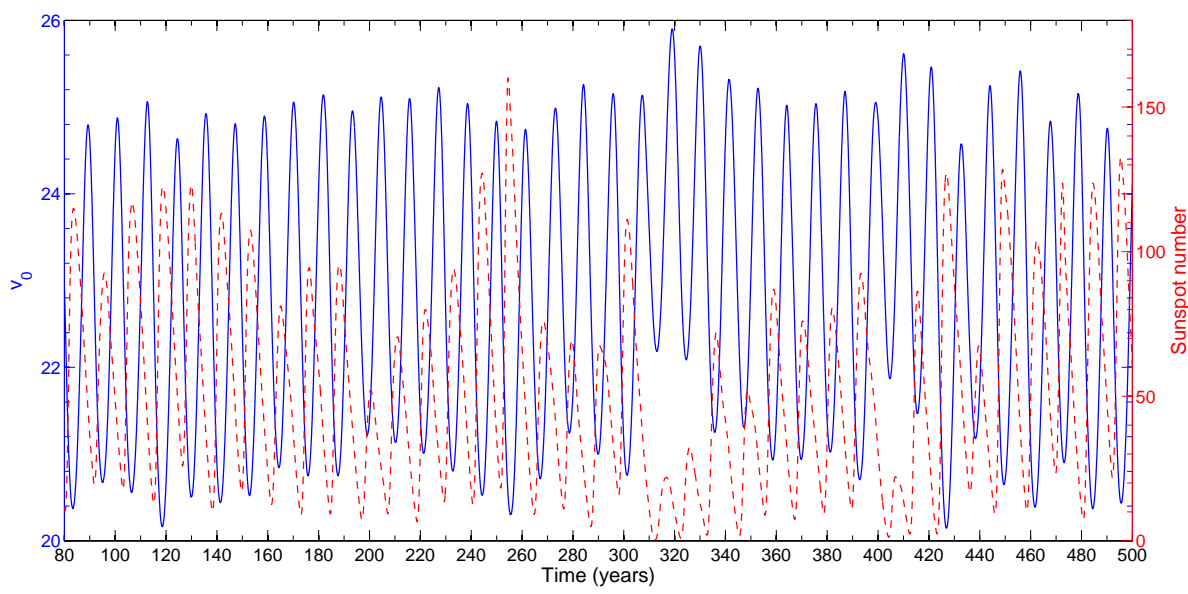

Figure 6. Results obtained by including stochastic fluctuations in the poloidal field generation process in the model of which results have been presented in Figure 5. Note that in this case we have plotted the sunspot eruptions (dashed line) instead of energy density of toroidal field as we have done earlier. The solid line shows the amplitude of meridional circulation.

magnitude larger. In the low-diffusivity situation, we found that the quenching of meridional circulation leads to an instability which ultimately reduces the meridional circulation to a very low value and the solution has a very large period. It is true that this instability can be suppressed by a sufficiently strong $\alpha$-quenching. However, we argue that such a strong $\alpha$-quenching is unphysical, making it unlikely that the solar dynamo is a low-diffusivity dynamo. On the other hand, when we run the DC99 model with a high diffusivity, we find it to be stable though the results do not look solar-like. To show the effect of the quenching of meridional circulation on a solar-like dynamo model with high diffusivity, we carry on some runs with the K10 model. In this case also, the solution is stable and the quenching of meridional circulation does not have a big effect on the dynamo except producing a period variation of the meridional circulation as observed. Our main conclusion is that a solar dynamo model with the quenching of the meridional circulation becomes unstable if the diffusivity is lower than about $2 \times 10^{11} \mathrm{~cm}^{2} \mathrm{~s}^{-1}$, unless there is also a strong $\alpha$-quenching to suppress this instability. Since $\alpha$-quenching in the real Sun is unlikely to be as strong as assumed by Dikpati and Charbonneau (1999), our study indicates that the solar dynamo is most probably a high-diffusivity dynamo. Several earlier authors already pointed out that the high-diffusivity model of the solar dynamo was much more successful in explaining such things as the parity of the solar magnetic fields (Chatterjee, Nandy and Choudhuri 2004; Hotta and Yokoyama 2010b; Kitchatinov and Olemskoy 2011), the hemispheric coupling (Chatterjee and Choudhuri 2006, Goel and Choudhuri 2009), the correlation between the polar field at the solar minimum and the sunspot number in the next cycle (Jiang, Chatterjee, and Choudhuri 2007), the Waldmeier effect (Karak and Choudhuri 2011), the Maunder minimum (Choudhuri and Karak 2009. 
Karak 2010), the periods and the amplitudes of the last 23 cycles (Karak 2010). Our present study further strengthens the case for the high-diffusivity dynamo.

The main effect of the quenching of meridional circulation in a high-diffusivity dynamo is that the meridional circulation varies with the solar cycle in a periodic way, becoming weaker at the time of the solar maximum. We have chosen the parameters of our model such that this periodic variation of the meridional circulation matches with what is reported by Hathaway and Rightmire (2010) from observations. In a recent work, Nandy, Munoz-Jaramillo and Martens (2011) have assumed that the meridional circulation changes randomly at every solar maximum, without having any correlation with the strength of the cycle. We disagree with this assumption and believe that the meridional circulation decreases at the solar maximum due to the Lorentz force of the magnetic fields in a systematic deterministic way. In this paper, we have treated the backreaction of the magnetic field on the meridional circulation in a very simplistic way through Equation (3). We have embarked on a more detailed calculation of this by solving the Navier-Stokes equation and hope to present the results of this calculation in a future paper.

\section{Acknowledgment}

We would like to thank the anonymous referee for valuable suggestions which helped us to improve the manuscript. This work is partly supported by DST through the J. C. Bose Fellowship awarded to ARC. BBK thanks CSIR, India for financial support.

\section{References}

Basu, S., Antia, H. M.: 2010, Astrophys. J., 717, 488.

Charbonneau, P.: 2010, Living Rev. Solar Phys., 7, 3.

Chatterjee, P., Choudhuri, A. R.: 2006, Solar Phys., 239, 29.

Chatterjee, P., Nandy, D., Choudhuri, A. R.: 2004, Astron. Astrophys., 427, 1019.

Choudhuri, A. R.: 1989, Solar Phys., 123, 217.

Choudhuri, A. R.: 1992, Astron. Astrophys., 253, 277.

Choudhuri, A. R.: 2011, Pramana, 77, 77.

Choudhuri A. R., Chatterjee P., Jiang J.: 2007, Phys. Rev. Lett., 98, 1103

Choudhuri, A. R., Karak, B. B. 2009, Res. Astron. Astrophys., 9, 953.

Choudhuri, A. R., Nandy, D., Chatterjee, P.: 2005, Astron. Astrophys., 437, 703.

Choudhuri, A. R., Schüssler, M., Dikpati, M.: 1995, Astron. Astrophys., 303, L29.

Dikpati, M., Charbonneau, P.: 1999, Astrophys. J., 518, 508.

Dikpati, M., Gilman, P. A.: 2006, Astrophys. J., 649, 498.

D'Silva, S., Choudhuri, A. R.: 1993, Astron. Astrophys., 272, 621.

Durney, B. R.: 1995, Solar Phys., 160, 213.

Goel, A., Choudhuri, A. R.: 2009, RAA, 9, 115.

Hathaway, D. H., Rightmire. L.: 2010, Sci., 327, 1350.

Hotta, H., Yokoyama, T.: 2010a, Astrophys. J., 709, 1009.

Hotta, H., Yokoyama, T.: 2010b, Astrophys. J., 714, L308.

Jiang, J., Chatterjee, P., Choudhuri, A. R.: 2007, MNRAS, 381, 1527.

Karak, B. B.: 2010, Astrophys. J., 724, 1021.

Karak, B. B., Choudhuri, A. R.: 2011, MNRAS, 410, 1503.

Kitchatinov, L. L., Ruediger, G.: 1995, Astron. Astrophys., 299, 446.

Kitchatinov, L. L., Olemskoy, S. V.: 2011, Solar Phys. (in press), arXiv:1108.3138 
Leighton, R. B.: 1969, Astrophys. J., 156, 1.

Nandy, D., Choudhuri, A. R. : 2002, Science, 296, 1671.

Nandy, D., Muñoz-Jaramillo, A., Martens, P. C. H.: 2011, Nature, 471, 80.

Parker, E. N.: 1955, Astrophys. J., 122, 293.

Parker, E. N.: 1979, Cosmical Magnetic Fields, Oxford University Press

Lopes, I., Passos, D.: 2009a, MNRAS, 397, 320.

Lopes, I., Passos, D.: 2009b, Solar Phys., 257, 1.

Passos, D., Lopes, I.: 2008, Astrophys. J., 686, 1420.

van Ballegooijen, A. A., Choudhuri, A. R.: 1988, Astrophys. J., 333, 965

Rempel, M.: 2006, Astrophys. J., 647, 662.

Yeates, A. R., Nandy, D., Mackay, D. H.: 2008, Astrophys. J., 673, 544. 